OPEN ACCESS

Edited by:

Ana Afonso,

University of São Paulo, Brazil

Reviewed by:

Mahmoud M. Taha,

Zagazig University, Egypt

Kalaimani Elango,

University of Nevada, Las Vegas,

United States

*Correspondence:

Oana Antal

antal.oanna@gmail.com

Specialty section:

This article was submitted to

Intensive Care Medicine and

Anesthesiology,

a section of the journal

Frontiers in Medicine

Received: 21 April 2021

Accepted: 19 October 2021

Published: 05 November 2021

Citation:

Trancă SD, Antal $O$ and Farcaş $A D$ (2021) Case Report: Acute Splenic Artery Thrombosis in a COVID 19

Postpartum Patient.

Front. Med. 8:698627.

doi: 10.3389/fmed.2021.698627

\section{Case Report: Acute Splenic Artery Thrombosis in a COVID 19, Postpartum Patient}

\author{
Sebastian Daniel Trancă ${ }^{1,2}$, Oana Antal ${ }^{1,3 *}$ and Anca Daniela Farcaş ${ }^{4,5}$ \\ ${ }^{1}$ Surgery Department, "Iuliu Haţieganu" University of Medicine and Pharmacy, Cluj-Napoca, Romania, ${ }^{2}$ Department of \\ Anaesthesia and Intensive Care, Emergency Clinical County Hospital, Cluj-Napoca, Romania, ${ }^{3}$ Department of Anaesthesia \\ and Intensive Care, Clinical Institute of Urology and Renal Transplantation, Cluj-Napoca, Romania, ${ }^{4}$ Internal Medicine \\ Department, "Iuliu Haţieganu" University of Medicine and Pharmacy, Cluj-Napoca, Romania, ${ }^{5}$ Cardiology Department, \\ Emergency Clinical County Hospital, Cluj-Napoca, Romania
}

The incidence of thromboembolic disease is reported to be high in SARS-CoV 2 disease. Pregnancy, an already physiologically hypercoagulable state, associated to COVID 19, generates even more concern regarding the potentially increased risk of thrombotic events. The exact incidence of such complications is yet unknown, but there is data suggesting that coagulopathy and thromboembolism are both increased in pregnancies affected by COVID-19. Since the outbreak of the COVID 19 pandemics, the most common described thrombotic events associated with SARS-COV2 infection have been venous thromboembolism and disseminated intravascular coagulation, while arterial thrombotic events are less commonly described. Splenic infarction is a rare disorder that can be secondary to a hypercoagulable state. There are only few cases of splenic infraction described, but none with splenic artery thrombosis, in a post-partum patient, on therapeutic anticoagulation regimen. We present the case of a 31-year-old Caucasian, 26 weeks pregnant woman, with no prior medical history, admitted to the hospital with a severe form of COVID 19 pneumonia and who, during the course of the disease, developed a massive splenic infarction with splenic artery thrombosis.

Keywords: splenic artery thrombosis, splenic infarction, post-partum, COVID 19 complications, case report

\section{INTRODUCTION}

Incidence of thromboembolic disease is reported to be high in SARS-CoV2 disease $(1,2)$. A multitude of organ systems can be affected by this life threatening complication (3).

We present the case of a 31-year-old, with life threatening COVID 19 pneumonia, who developed splenic artery thrombosis with a massive splenic infarction while being therapeutically anticoagulated.

\section{CASE DESCRIPTION}

A 31-year-old Caucasian, 26 weeks pregnant woman, with a BMI of 29, with no prior medical history, was admitted to the hospital with progressive dyspnea, fatigue, and dry cough, which started 5 days prior to hospital admission. Real-Time PCR tested positive for SARS-CoV-2 and CT scan showed COVID 19 pneumonia in $90 \%$ of the lung. Five days after admission she was 


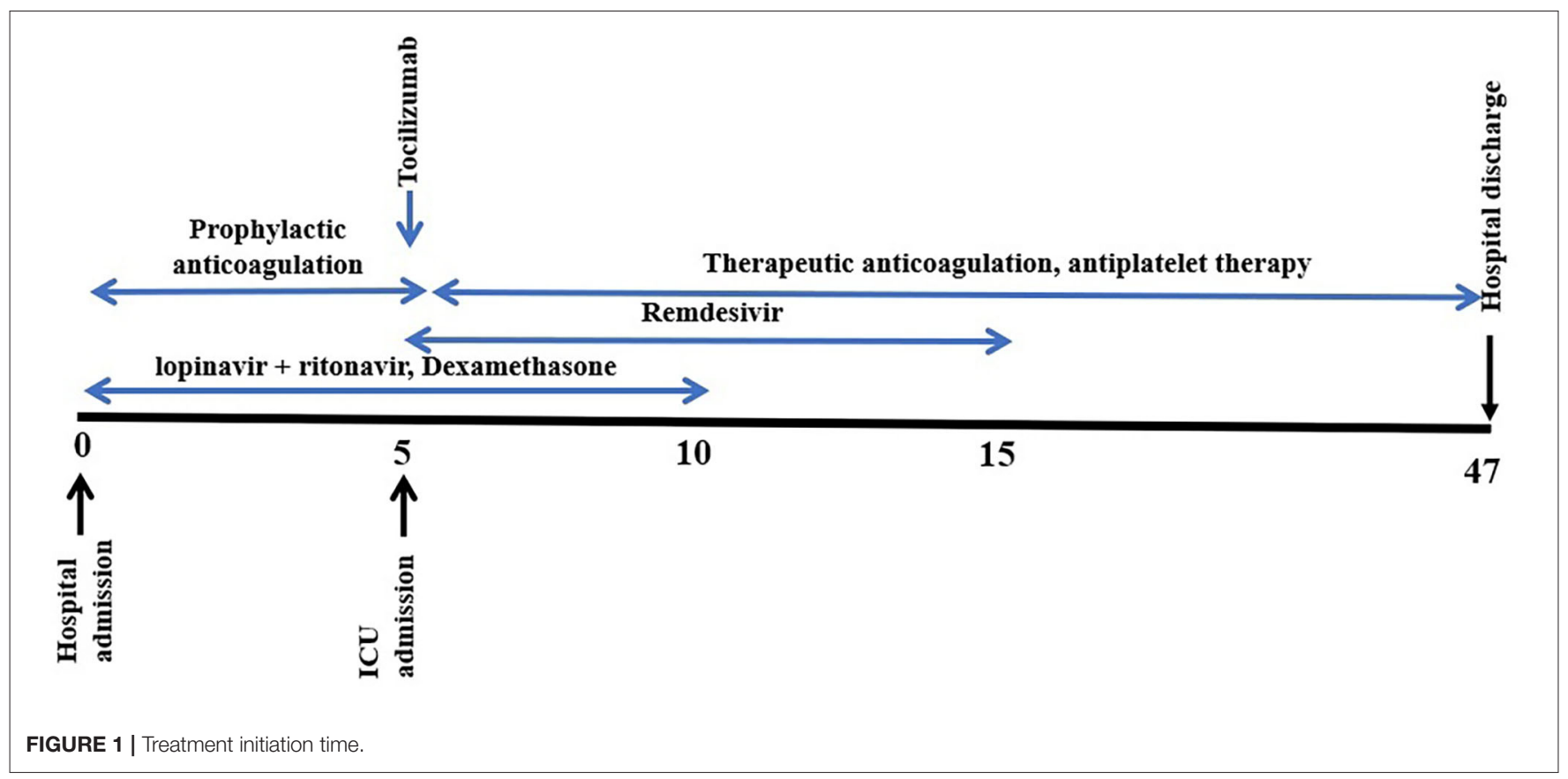

transferred to the ICU for severe dyspnea and polypnea, with extreme hypoxemia, where shortly afterwards, she was intubated and mechanically ventilated. Respiratory parameters on controlled mechanical ventilation and continuous neuromuscular blocking agents (NMBA) administration showed severe hypoxemia, with a $\mathrm{PaO}_{2} / \mathrm{FiO}_{2}$ ratio of 78 , with low compliance. The respiratory course of the disease was complicated by two episodes of tension pneumothorax, adequately drained, and ventilator associated pneumonia (VAP) treated according to cultures. Following treatment, the pulmonary status improved gradually, reaching extubation criteria 16 days after the initial intubation. During admission time the cardiac function was within normal range; vasopressor support was necessary during the septic episodes, in order to maintain a MAP $>70 \mathrm{~mm} \mathrm{Hg}$. The renal function remained normal. After extubation, the patient showed normal cognitive functions, but had signs of severe neuropathy and myopathy.

A 10-days course of Remdesivir was initiated at day 5 after admission, alongside Dexamethasone (started at the time of admission); therapeutic systemic anticoagulation with Enoxaparin $(1 \mathrm{mg} / \mathrm{kg}$, twice a day) and antiplatelet therapy (Aspirin) was also initiated at ICU admission time. Tocilizumab was also administered during early admission days, after an increased IL6 value was observed (5 days after admission). A 10days course of lopinavir + ritonavir was initiated at the time of hospital admission (Figure 1).

The initially daily pregnancy follow up showed fetus viability, but with a gradually decreased umbilical cord blood flow. Ten days after her hospital admission, the intrauterine death of the fetus was revealed; a cesarean section was performed.

In Table 1 and Figure 2, here are the dynamics of lab test during hospitalization.
During the course of the disease, she complained of mild, dull abdominal pain while developing progressive thrombocytosis; the clinical examination showed a soft, non-distended, nontender abdomen. The CT-scan confirmed a 75\% splenic infarction, with splenic artery thrombosis, with a major reduction in blood flow (Figure 3).

We would like to emphasize, that during her hospital stay, the patient was continuously on therapeutic anticoagulation (considered a high risk patient due to pregnancy and intrauterine death of the fetus) with mildly elevated D-dimer values, and fibrinogen levels within normal range, with the exception 1 week, when values ranged between 9 and $5 \mathrm{~g} / \mathrm{L}$. There were no thromboembolic or infarctions noted on a prior CT chest and abdomen done earlier in the admission period. Both the therapeutic anticoagulation with LMWH and the antiplatelet therapy were continued after the diagnosis of the thrombotic event.

\section{DISCUSSION}

Although initially described as a viral pneumonia, SARS-CoV2 infection seems to present features of a multisystem disease, with impairment of several organs (4). Splenic infarction is a rare disorder that can be secondary to a hypercoagulable state (5). COVID-19 was associated with hypercoagulability and increased risk for venous and arterial thromboembolism (1-3). The underlying mechanisms of thrombo-embolic events during COVID 19 associated critical illness are not yet fully understood. The incidence of such complications was reported to be as high as $69 \%$, in critically ill COVID 19, intubated and mechanically ventilated patients, under prophylactic or therapeutic anticoagulant dosage (6). Coagulation abnormalities 
TABLE 1 | Relevant laboratory findings during hospital stay.

\begin{tabular}{|c|c|c|c|c|c|c|}
\hline & $\begin{array}{l}\text { 1st week after } \\
\text { admission }\end{array}$ & $\begin{array}{l}\text { 2nd week after } \\
\text { admission }\end{array}$ & $\begin{array}{l}\text { 3rd week after } \\
\text { admission }\end{array}$ & $\begin{array}{l}\text { 4th week after } \\
\text { admission }\end{array}$ & $\begin{array}{l}\text { 5th week after } \\
\text { admission }\end{array}$ & $\begin{array}{l}\text { 6th week after } \\
\text { admission }\end{array}$ \\
\hline $\begin{array}{l}\text { Ferritin } \mathrm{mg} / \mathrm{dl} \text { (normal range: male } 12-300 \mathrm{ng} / \mathrm{ml} \text {, } \\
\text { female: } 10-150 \mathrm{ng} / \mathrm{ml} \text { ) }\end{array}$ & & 363 & 261 & 205 & 264 & 135 \\
\hline$\|_{6} \mathrm{pg} / \mathrm{ml}$ (normal range: < 7 pg/ml) & & 339.48 & & & & \\
\hline CRP mg/L (normal range: 9-10 mg/L) & 3 & 435.7 & 218.9 & 36.3 & 72.4 & 33.1 \\
\hline PCT ng/L (normal range: <0.05 ng/L) & 0,1 & 3.4 & 0.2 & 10.61 & 0.06 & 0.35 \\
\hline Leucocytes $10^{9} / L$ (normal range: $4-9 \times 10^{9}$ ) & 10,6 & 50.04 & 25.77 & 18.26 & 18.4 & 17.2 \\
\hline $\begin{array}{l}\text { Thrombocytes } 10^{9} / L \text { (normal range: } 150-450 \times \\
10^{9} \text { ) }\end{array}$ & 335 & 261 & 902 & 721 & 484 & 565 \\
\hline Fibrinogen g/L (normal range: 2-4 g/L) & & 7.77 & 4.8 & 2.34 & 3.35 & 3.63 \\
\hline aPTT sec (normal range: $24,5-36,5 \mathrm{~s}$ ) & & 24.1 & 24 & 26.4 & 23.3 & 24.9 \\
\hline
\end{tabular}

aPTT, activated partial thromboplastin time; CRP, C-reactive protein; II6, interleukin 6; PCT, procalcitonin; PT, prothrombin time.

A

Ferritin and CRP

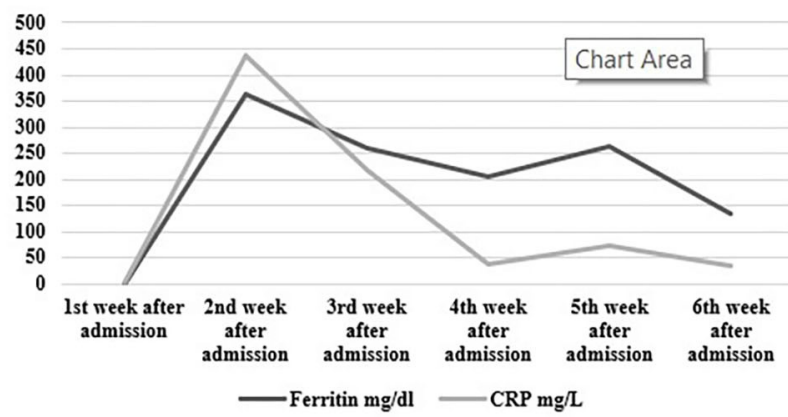

C

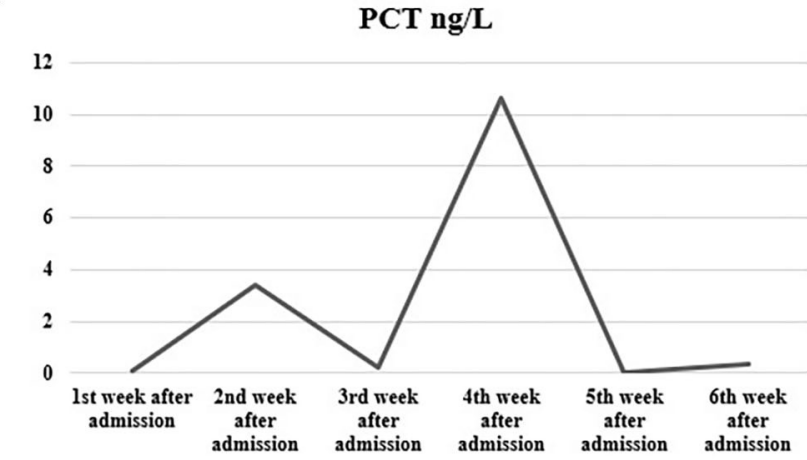

B

Fibrinogen $\mathbf{g} / \mathbf{L}$

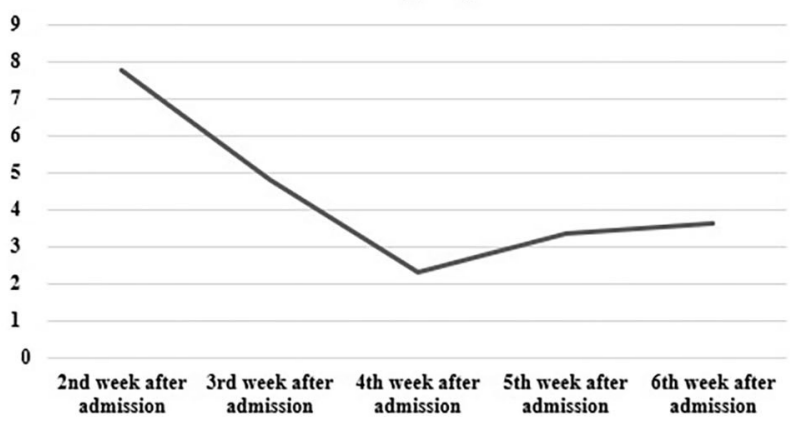

D Thrombocytes 109/L

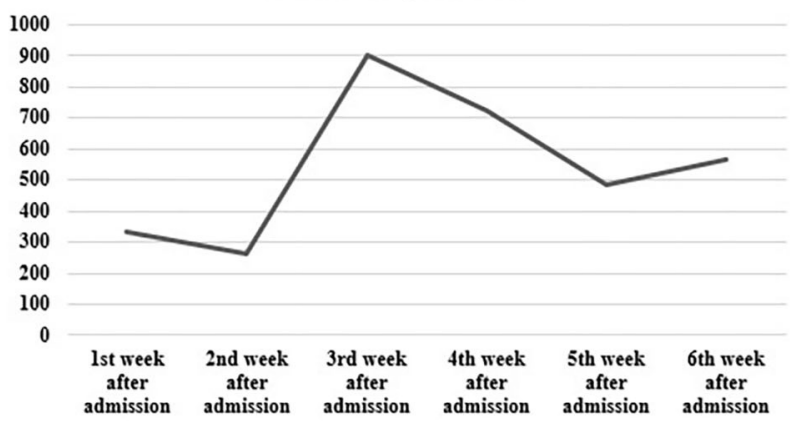

FIGURE 2 | Dynamics of (A) C-reactive protein (CRP) and Ferritin, (B) fibrinogen, (C) procalcitonin (PCT), and (D) thrombocytes during the course of the disease.

may have a major impact on the outcome of COVID-19 patients (7).

Since the outbreak of the pandemics, the most common described thrombotic events associated with SARS-COV2 infection have been venous thromboembolism, but large-vessel ischemic stroke, acute upper or lower limb ischemia, abdominal and thoracic aortic thrombosis, mesenteric ischemia, myocardial infarction, and disseminated intravascular coagulation, have also been reported (8-14).

A recent systematic review on arterial thrombosis (AT) in COVID 19 patients, showed that most of the patients presenting AT were male, elderly, and had comorbidities (15). 

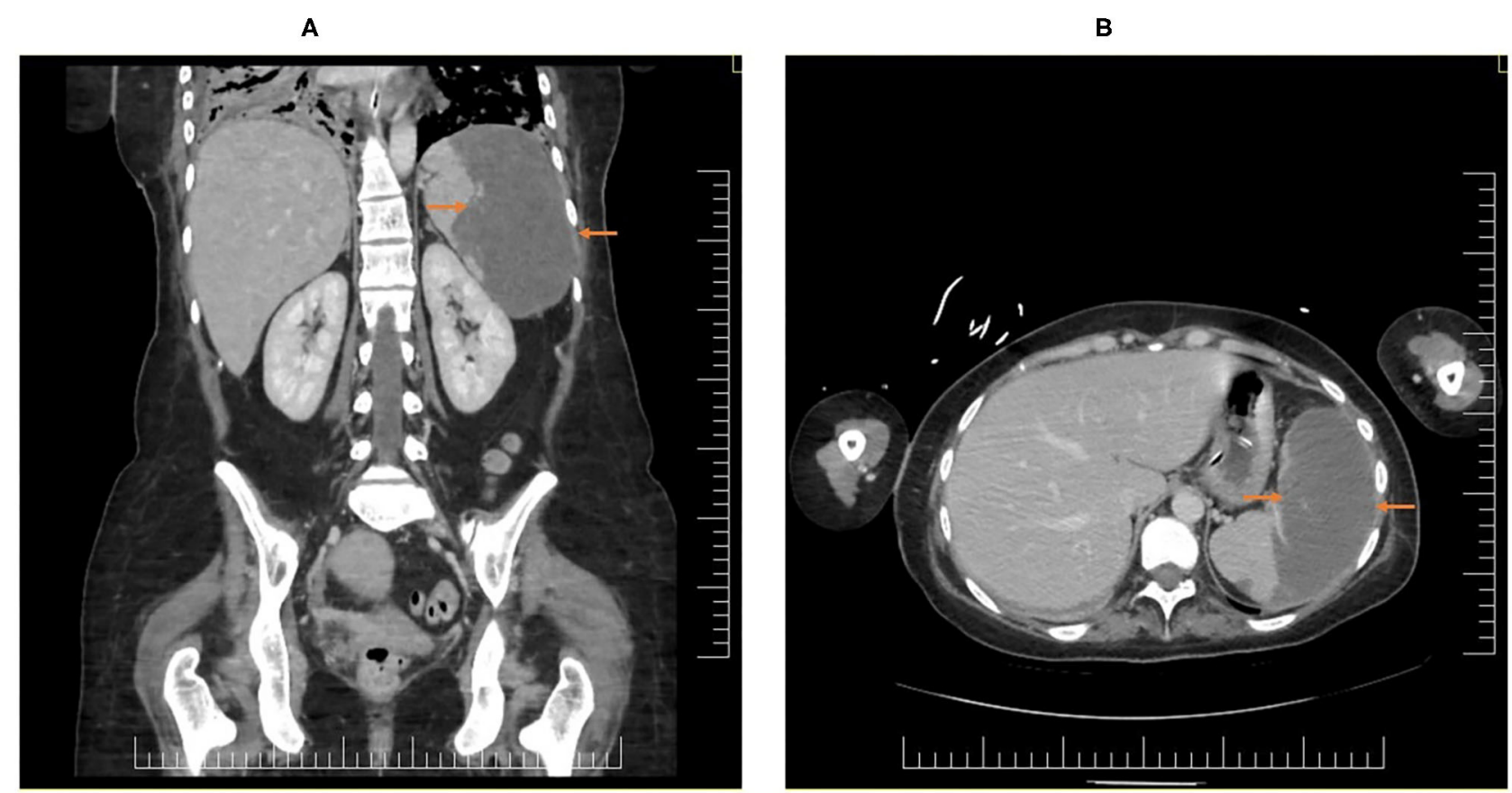

FIGURE 3 | CT scan images showing the splenic infarction, (A) coronal plane and (B) axial plane.

The anatomical distribution of arterial thrombotic events was shown to be wide, but none involved the splenic artery. The particularity of this case report relies on the fact that our patient was a young, healthy women, in post-partum period with an unusual site for AT.

While pregnancy is already a physiologically hypercoagulable state, there is concern regarding the potentially increased risk of thrombotic complications. The exact incidence is yet unknown, but there is data suggesting that coagulopathy and thromboembolism are both increased in pregnancies affected by COVID-19 (16). There are only few cases of splenic infarction described, but none with splenic artery thrombosis, in a post-partum, otherwise healthy patient, on therapeutic anticoagulation regimen. The described case highlight splenic infarction, with splenic artery thrombosis as a thrombotic complication of COVID-19.

Regarding the anticoagulation therapy after the diagnosis of the thrombotic event, we decided to continue the therapeutic dose of LMWH as prescribed by the hospital's cardiologist. This decision was made based on the recent COVID 19 guidelines on anticoagulation in VTE and PE (17), and also on the fact that there were no contraindication to LMWH.

\section{REFERENCES}

1. Klok FA, Kruip M, van der Meer N, Arbous MS, Gommers D, Kant $\mathrm{KM}$, et al. Incidence of thrombotic complications in critically ill ICU

\section{CONCLUSION}

COVID-19 disease may be associated with AT in unusual sites, such as the splenic artery, even in patients on therapeutic anticoagulation.

\section{DATA AVAILABILITY STATEMENT}

The original contributions presented in the study are included in the article/supplementary material, further inquiries can be directed to the corresponding author/s.

\section{ETHICS STATEMENT}

Ethical review and approval was not required for the study on human participants in accordance with the local legislation and institutional requirements. The patients/participants provided their written informed consent to participate in this study.

\section{AUTHOR CONTRIBUTIONS}

All authors listed have made a substantial, direct and intellectual contribution to the work, and approved it for publication.

patients with COVID-19. Thromb Res. (2020) 191:145-7. doi: 10.1016/ j.thromres.2020.04.013

2. Klok FA, Kruip M, van der Meer N, Arbous MS, Gommers D, Kant KM, et al. Confirmation of the high cumulative incidence of thrombotic complications 
in critically ill ICU patients with COVID-19: an updated analysis. Thromb Res. (2020) 191:148-50. doi: 10.1016/j.thromres.2020.04.041

3. Mondal S, Quintili AL, Karamchandani K, Bose S. Thromboembolic disease in COVID-19 patients: a brief narrative review. J Intensive Care. (2020) 8:70. doi: 10.1186/s40560-020-00483-y

4. Robba C, Battaglini D, Pelosi P, Rocco PRM. Multiple organ dysfunction in SARS-CoV-2: MODS-CoV-2. Expert Rev Respir Med. (2020) 14:865-8. doi: $10.1080 / 17476348.2020 .1778470$

5. O'Donnell M, Shatzel JJ, Olson SR, Daughety MM, Nguyen KP, Hum J, et al. Arterial thrombosis in unusual sites: a practical review. Eur J Haematol. (2018) 101:728-36. doi: 10.1111/ejh.13165

6. Llitjos JF, Leclerc M, Chochois C, Monsallier JM, Ramakers M, Auvray M, et al. High incidence of venous thromboembolic events in anticoagulated severe COVID-19 patients. J Thromb Haemost. (2020) 18:1743-6. doi: $10.1111 /$ jth.14869

7. Robba C, Battaglini D, Ball L, Valbusa A, Porto I, Della Bona R, et al. Coagulative disorders in critically ill COVID-19 patients with acute distress respiratory syndrome: a critical review. J Clin Med. (2021) 10:140. doi: $10.3390 / \mathrm{jcm} 10010140$

8. Griffin DO, Jensen A, Khan M, Chin J, Chin K, Parnell R, et al. Arterial thromboembolic complications in COVID-19 in low-risk patients despite prophylaxis. Br J Haematol. (2020) 190:e11-3. doi: 10.1111/bjh. 16792

9. Beccara LA, Pacioni C, Ponton S, Francavilla S, Cuzzoli A. Arterial mesenteric thrombosis as a complication of SARS-CoV-2 infection. Eur J Case Rep Intern Med. (2020) 7:001690. doi: 10.12890/2020_001690

10. Gomez-Arbelaez D, Ibarra-Sanchez G, Garcia-Gutierrez A, ComangesYeboles A, Ansuategui-Vicente M, Gonzalez-Fajardo JA. COVID-19-related aortic thrombosis: a report of four cases. Ann Vasc Surg. (2020) 67:10-13. doi: 10.1016/j.avsg.2020.05.031

11. Driggin E, Madhavan MV, Bikdeli B, Chuich T, Laracy J, Biondi-Zoccai G, et al. Cardiovascular considerations for patients, health care workers, and health systems during the COVID-19 pandemic. J Am Coll Cardiol. (2020) 75:2352-71. doi: 10.1016/j.jacc.2020.03.031

12. Bridwell R, Long B, Gottlieb M. Neurologic complications of COVID-19. Am J Emerg Med. (2020). 38:1549.e3-7. doi: 10.1016/j.ajem.2020.05.024
13. Kalpatthi R, Kiss JE. Thrombotic thrombocytopenic purpura, heparininduced thrombocytopenia, and disseminated intravascular coagulation. Crit Care Clin. (2020) 36:357-77. doi: 10.1016/j.ccc.2019.12.006

14. Avila J, Long B, Holladay D, Gottlieb M. Thrombotic complications of COVID-19. Am J Emerg Med. (2021) 39:213-8. doi: 10.1016/ j.ajem.2020.09.065

15. Cheruiyot I, Kipkorir V, Ngure B, Misiani M, Munguti J, Ogeng'o J. Arterial thrombosis in coronavirus disease 2019 patients: a rapid systematic review. Ann Vasc Surg. (2021) 70:273-81. doi: 10.1016/j.avsg.2020.08.087

16. Servante J, Swallow G, Thornton JG, Myers B, Munireddy S, Malinowski AK, et al. Haemostatic and thrombo-embolic complications in pregnant women with COVID-19: a systematic review and critical analysis. BMC Pregnancy Childbirth. (2021) 21:108. doi: 10.1186/s12884-021-03568-0

17. Moores LK, Tritschler T, Brosnahan S, Carrier M, Collen JF, Doerschug K, et al. Prevention, diagnosis, and treatment of VTE in patients with coronavirus disease 2019: chest guideline and expert panel report. Chest. (2020) 158:114363. doi: 10.1016/j.chest.2020.05.559

Conflict of Interest: The authors declare that the research was conducted in the absence of any commercial or financial relationships that could be construed as a potential conflict of interest.

Publisher's Note: All claims expressed in this article are solely those of the authors and do not necessarily represent those of their affiliated organizations, or those of the publisher, the editors and the reviewers. Any product that may be evaluated in this article, or claim that may be made by its manufacturer, is not guaranteed or endorsed by the publisher.

Copyright (c) 2021 Trancă, Antal and Farcaș. This is an open-access article distributed under the terms of the Creative Commons Attribution License (CC BY). The use, distribution or reproduction in other forums is permitted, provided the original author(s) and the copyright owner(s) are credited and that the original publication in this journal is cited, in accordance with accepted academic practice. No use, distribution or reproduction is permitted which does not comply with these terms. 